When to Pick the Losers:

Do Sentiment Indicators Improve Dynamic Asset Allocation?

\author{
DEVRaJ BASU ${ }^{1}$ \\ CHI-Hsiou $\mathrm{HunG}^{2}$ \\ Roel OOMEN ${ }^{1}$ \\ Alexander Stremme ${ }^{1}$ \\ ${ }^{1}$ Warwick Business School, University of Warwick \\ ${ }^{2}$ Durham Business School, University of Durham
}

FEBRUARY 2006

address for correspondence:

\author{
Alexander Stremme \\ Warwick Business School \\ University of Warwick \\ Coventry, CV4 7AL, United Kingdom \\ Tel: $+44(0) 2476522066$ \\ Email: alex.stremme@wbs.ac.uk
}




\title{
WHEN TO PICK THE LOSERs: \\ Do Sentiment Indicators Improve Dynamic Asset Allocation?
}

\author{
FEBRUARY 2006
}

\begin{abstract}
Recent finance research that draws on behavioral psychology suggests that that investors systematically make errors in forming expectations about asset returns, and thus that investor sentiment can have predictive power for asset returns. A number of empirical studies using both market and survey data as proxies for investor sentiment have found support for these theories. In this study we investigate whether investor sentiment as measured by a component of the University of Michigan survey can help improve dynamic asset allocation over and above the improvement achieved based on commonly used business cycle indicators. We find that the addition of sentiment variables to business cycle indicators considerably improves the performance of dynamically managed portfolio strategies, both for a standard markettimer as well as for a momentum-type investor. We find that sentiment-based dynamic trading strategies, even out-of-sample, would not have incurred any significant losses during the October 1987 crash or the collapse of the 'dot.com' bubble in late 2000. In contrast, standard business cycle indicators fail to predict these events, so that investors relying on these variables alone would have incurred significant losses. Interestingly however, strategies based only on sentiment do not perform well at all. It is the interaction between business cycle indicators and sentiment that makes the strategies work. These strategies are 'active alpha' strategies with low betas and high alphas, in contrast to business cycle based strategies which are effectively 'index-trackers' with high betas and considerably lower alphas.
\end{abstract}

JEL Classification: C32, F39, G11, G12 
"Modern markets show considerable micro efficiency ... I [hypothesize] considerable macro inefficiency, in the sense of long waves in the time series of aggregate indexes of security prices below and above various definitions of fundamental values."

Paul Samuelson, quoted in Shiller (2000), Irrational Exuberance.

\section{Introduction}

Recent finance research invokes investor sentiment, which reflects cognitive heuristics, to suggest that investors systematically make errors in forming expectations about asset returns. These errors are likely to cause significant mis-pricing in the short run, as documented for example by Shleifer (2000), and Hirshleifer (2001). The subsequent reversal of security prices to their fundamental levels then implies that measures of investor sentiment are likely to be correlated with stock returns. Fisher and Statman (2000), and Charoenrook (2005), among others, find evidence that such measures indeed predict stock returns.

In this paper, we investigate whether the predictive information contained in a specific set of investor sentiment indicators can be used to improve dynamic asset allocation. We test both simple market-timing strategies as well as strategies that allow the investor to allocate funds between two momentum portfolios ('winners' and 'losers'). We focus in particular on two main aspects; first we investigate if sentiment indicators can help avoid losses in market crashes or reduce portfolio risk during periods of high volatility. Second, we study whether sentiment is useful in active 'alpha-management', i.e. we ask if dynamic strategies can consistently 'beat the market' in the long run. The answer to both questions is affirmative: first, even out-of-sample our strategies would not have incurred any losses during the October 1987 crash or the collapse of the internet bubble in 2000. Second, in the long-run our strategies achieve consistent out-performance with alphas in excess of $15 \%$. The improvement in performance is statistically significant in all cases. 
Behavioral theories draw evidence from experimental psychology, which shows that individuals tend to form beliefs that are inconsistent with the economic paradigm of rational expectations. DeBondt and Thaler (1985) cite over-reaction in explaining return predictability based on long-term past returns. Their explanation is motivated by the observation that individuals tend to misinterpret observed events as representative descriptions of the population moments, while underestimating the randomness of observed outcomes (Kahneman and Tversky 1973). Investors mistakenly extrapolate extreme news events into the future, causing them to irrationally update their beliefs, thus leading to over-reaction.

On the other hand, Barberis, Shleifer, and Vishny (1998) rely on the conservatism bias (Edwards 1968) to motivate under-reaction. Because individuals tend to be excessively conservative in updating their expectations, securities prices do not adjust instantaneously to the arrival of new information. This price 'stickyness' leads to trends in returns over short horizons and hence might be a factor in explaining the momentum effect, first documented by Jegadeesh and Titman (1993). In contrast, Daniel, Hirshleifer, and Subrahmanyam (2001) focus on the self-attribution bias. As Shefrin (1999) writes (page 101), "self-attribution bias occurs when people attribute successful outcomes to their own skill but blame unsuccessful outcomes on bad luck." In the short run, information arrival will lead investors to become over-confident and result in over-reaction. The continued flow of information will eventually drive prices back to their fundamental levels, thus creating short-run predictability.

Recent empirical studies using direct measures ${ }^{1}$ of investor sentiment provide some evidence for these theories. Fisher and Statman (2000) find that investor sentiment is a reliable contrarian predictor of S\&P 500 returns, and Charoenrook (2005) documents the predictive power of the University of Michigan Consumer Sentiment index. Amromin and Sharpe (2005), using similar data, find that returns over medium horizons appear to be extrapolated from past returns, providing evidence for the under-reaction hypothesis.

\footnotetext{
${ }^{1}$ As opposed to market-based measures such as, for example, the closed-end fund discount, book-to-market ratios, decisions to issue stock rather than bonds.
} 
In this paper we use changes in specific components of the University of Michigan Consumer Sentiment survey data as a measure of individual investor sentiment. The Michigan survey is closely watched by economists and investors who believe it to convey information relevant to the stock market ${ }^{2}$. We focus here on that component of the index that measures consumers' perceptions of current business conditions and their predictions of business conditions in the near future, as this component seems to contain information most directly relevant to the stock market. We also include 3 and 6 month lags of these variables to see whether revisions in forecasts or perceptions, or the match (or mismatch) between past forecasts and current perceptions, also matter. Our approach thus differs from that in Charoenrook (2005), who uses the information in all the other components of the survey. As both theory and empirical evidence suggest that the predictive content of investor sentiment is not significantly related to the business cycle, we analyze whether sentiment indicators can improve the performance of market-timing strategies over and above the improvement achieved bases on commonly used macro-economic variables ${ }^{3}$, which are known to be correlated with the business cycle.

We first investigate whether out-of-sample, investor sentiment can predict extreme market movements and thus help avoid losses in market crashes or periods of high volatility. We find that sentiment-based dynamic trading strategies would not have incurred any significant losses during the October 1987 crash or the collapse of the 'dot.com' bubble in late 2000 . In contrast, standard business cycle indicators fail to predict these events, so that investors relying on these variables alone would have incurred significant losses. A possible explanation is that strategies based on macro variables typically have very high betas, which amplify market movements in either direction. Thus, these are 'good-weather-strategies', making good gains in bull markets but incurring excessive losses in market downturns. In contrast, sentiment indicators seem to allow asset managers to 'de-couple' their portfolios from the business cycle (with betas between 0.2 and 0.65 ) and thus successfully time the market.

\footnotetext{
${ }^{2}$ Some articles in the financial press claim that the survey actually moves rather than predicts markets.

${ }^{3}$ We use the short rate, the slope of the Treasury yield curve, as well as the credit yield spread.
} 
In addition to pure market-timing strategies, we also consider strategies that allow the investor to allocate funds between two momentum portfolios ('winners' and 'losers') based on past performance. While momentum investing improves performance relative to pure market-timing, we also find an interesting difference in the behavior of portfolio weights. While market-timing strategies are forced to move in and out of the market, often taking short or leveraged positions, we find that momentum strategies behave much more like hedge funds: in periods of market turmoil, they remain fully invested in the risk-free asset while taking spread (long-short) positions in the momentum portfolios. In bull markets, they remain heavily invested in the winner stocks, while taking spread positions between losers and the risk-free asset. Interestingly, momentum strategies based on business cycle variables alone show a very different behavior. In most cases, they would 'ignore' the losers and simply time the market with the winner portfolio.

When we analyze the long-run performance of the different types of strategies, an interesting pattern emerges. Consistent with our earlier results, business cycle variables on their own are not sufficient to achieve consistently successful market-timing ${ }^{4}$. Strategies based on these variables (even when allowed to pick between winners and losers) simply track the index with betas in excess of 1 , thus amplifying any market movement. In contrast, the addition of sentiment indicators leads to significantly higher performance (with alphas increasing from $3-5 \%$ to more than 15\%). For the same target return, efficient risk-control strategies based on business cycle variables still have a volatility of $14 \%$, while those using sentiment decrease volatility to about $7 \%$. Interestingly however, strategies based only on sentiment do not perform well at all. It is the interaction between business cycle indicators and sentiment that makes the strategies work.

To assess the statistical significance of our results, we use a test developed in Abhyankar, Basu, and Stremme (2005). This test is based on the difference in the slope of the efficient

\footnotetext{
${ }^{4}$ Although, as noted in Kandel and Stambaugh (1996), timing the market correctly only once in 100 times can still generate significant abnormal returns.
} 
frontiers with and without the optimal use of predictive information. As this test has a known statistical distribution (both in finite sample as well as asymptotically), we can assess whether the expansion of the frontier due to predictability is statistically significant or just a product of sampling error. We find that the improvement due to the use of sentiment variables is significant (at the $5 \%$ level in all cases, in some cases at the $1 \%$ level), while business cycle variables alone only lead to insignificant performance gains (with $p$-values around 20-30\%).

We draw three main conclusions from our empirical findings. First our out-of-sample experiment shows that the addition of sentiment variables to business cycle indicators considerably improves the performance of dynamically managed portfolio strategies, both for a standard market-timing as well as momentum-investing. In contrast, strategies based on the business cycle variables alone only slightly out-perform buy-and-hold strategies and under-perform the market index in several cases. Second, our in-sample test shows that these results are statistically significant and not just artifacts of the chosen sub-period. Finally, the strategies based on the sentiment variables are 'active alpha' strategies with low betas and high alphas, in contrast to business cycle based strategies which are effectively 'index-trackers' with high betas with considerably lower alphas.

The remainder of this paper is organized as follows; In Section 2, we describe the data we use and our empirical methodology. The results of our empirical analysis are reported in Section 3. Section 4 concludes. Detailed descriptions of the portfolio strategies used in this study, and the measures and tests used to assess their performance, are given in the Appendix.

\section{Data and Methodology}

In this section we describe the predictive instruments and base assets used in our empirical analysis, as well as the portfolio strategies and the performance measures used to evaluate our results. We give here only an intuitive description, details can be found in Appendix A. 


\subsection{Data}

For our empirical analysis, we use monthly data covering the period from January 1980 until December 2003. The choice of sample period is mainly dictated by the availability of the predictive instruments we wish to use.

\section{SENTIMENT INDICATORS}

Each month, the Survey Research Center at the University of Michigan conducts a minimum of 500 phone interviews, which are used for the computation of a number of commonly cited gauges of the economy, such as the Index of Consumer Sentiment. The Michigan survey questions include several inquiries regarding the respondents perception of business and financial conditions, as well as their own economic prospects, over horizons varying from one to five years. Among the questions we focus here on two that pertain to consumer's expectations of current business conditions and business conditions in 6 months. The responses can be currently good, bad or normal and better, worse or normal in 6 months and the we focus on the percentage of respondents who think current business conditions are good minus those who think it is bad ('net good', NG) and similarly percentage of those who think it will be better in 6 months minus those who think it will be worse ('net better', NB). We also include the 3 and 6 month lags of each of these variables (NB3L, NG3L, NB6L, and NG6L). These data have been available at monthly frequency since 1978. Our sample period begins in January 1980 (to accommodate all required lags of the instruments) and ends in December 2003.

\section{Business Cycle Predictors}

The business cycle predictors we use are the 1-month US Treasury bill rate (TB1M), which has been shown to be a proxy for future economic activities (Fama and Schwert 1977), the term spread (TSPR, defined as the difference in yield on the 10-year and 1-year Treasury bond), which has been shown to be closely related to short-term business cycles (Fama and French 1988), and the credit spread (CSPR, the difference in yield between a 10-year 
AAA-rated corporate bond and the corresponding Treasury bond), which tracks long term business cycle conditions (Fama and French 1988).

\section{INVESTIBLE ASSETS}

The base assets are the 1-month Treasury bill and, in case of the market-timing strategies, the CRSP value-weighted index. For the momentum selection strategies, we replace the index by two portfolios sorted on past returns ('winners' and 'losers'). To construct these, monthly US dollar-denominated stock returns (including dividends and capital gains) are obtained from Datastream. The sample covers 4,291 firms in the US from January 1980 to December 2003. Both listed and de-listed firms are included to control for survivorship bias. At each re-balancing point we sort stocks based on their past six-month compounded returns, and form 10 value-weighted momentum decile portfolios. The returns on these portfolios are computed for the six months following the re-balancing. The 'winner' and 'loser' portfolios (M01 and M10) contain stocks within the highest lowest decile of past returns. The formation of momentum portfolios is non-overlapping thus reducing trading frequencies and hence transaction costs implicit in the portfolio construction.

\subsection{Dynamically Efficient Trading Strategies}

Most of the existing literature on predictability and market-timing focuses on 'myopically optimal' strategies. The portfolio weights of such strategies are chosen ex-post, based on the conditional moments of asset returns. In contrast, we focus here on 'dynamically optimal', i.e. unconditionally efficient strategies, as studied in Ferson and Siegel (2001), and Abhyankar, Basu, and Stremme (2005). While the portfolio weights of the former are determined ex-post on the basis of the conditional return moments, the weights of the latter are determined exante as functions of the predictive instruments. In this sense, dynamically optimal strategies are truly actively managed, while myopically optimal strategies can be though of as sequences of one-step-ahead efficient static portfolios. Because dynamically optimal strategies are designed to be efficient with respect to their long-run unconditional moments, they display a 
more 'conservative' response to changes in the predictive instruments ${ }^{5}$. This is an important consideration in particular with respect to transaction costs.

Studies that have examined market-timing using predictive variables such as the short rate (Breen, Glosten, and Jagannathan 1989), or time-variation in the conditional Sharpe ratio Whitelaw (2005), have employed naive portfolio strategies related to conditional efficiency. In contrast, unconditionally efficient strategies optimally utilize both the predictive information and the time-variation in the conditional Sharpe ratio (Cochrane 1999), and can significantly outperform naive market-timing strategies.

We provide precise specifications of the weights of dynamically efficient strategies in Appendix A.1. In our empirical applications, we consider both efficient minimum-variance strategies (designed to track a given target average return), as well as efficient maximumreturn strategies (designed to track a given target volatility). The former are particularly useful in risk management as they provide portfolio insurance against crashes and periods of excess volatility. The latter can be though of as 'active alpha' strategies, designed to achieve maximum performance at a tolerable level of risk.

\subsection{Measuring the Value of Return Predictability}

We employ a variety of performance criteria to measure the gains due to the optimal use of return predictability. To assess the long-run performance of dynamically managed strategies based on business cycle and sentiment indicators, we use a variety of standard ex-post portfolio performance measures. These include Sharpe ratios, Jensen's alpha, and information ratios.

\footnotetext{
${ }^{5}$ See also Ferson and Siegel (2001) or Abhyankar, Basu, and Stremme (2005).
} 


\section{Measures of Statistical Significance}

To capture the incremental gains due to the optimal use of predictive information, we compare the performance of optimally managed portfolios with that of traditional 'fixed-weight' strategies, i.e. those for which the weights $\theta_{k}$ do not depend on the instruments $\left.Z_{t-1}\right)$. We wish measure the extent to which the optimal use of predictive information expands the efficient frontier, and hence the opportunity set available to the investor. Because the location of the global minimum-variance (GMV) portfolio is virtually unaffected by the introduction of predictive variables (see also Figure 3), we use the asymptotic slope of the frontier (i.e. the Sharpe ratio relative to the zero-beta rate associated with the mean of the GMV) as such a measure. Because we can show that the difference in (squared) slopes of the frontiers with and without the optimal use of predictability has a known $\left(\chi^{2}\right)$ distribution, we are able to assess the statistical significance of any gains due to predictability. A precise definition of our test statistic is given in Appendix A.2.

\section{Measures of Economic Value}

In addition to our statistical tests, we also employ a utility-based framework to assess the economic value of return predictability. Following Fleming, Kirby, and Ostdiek (2001), we consider a risk averse investor whose preferences over future wealth are given by a quadratic von Neumann-Morgenstern utility function. Consider now an investor who faces the decision whether or not to acquire the skill and/or information necessary to implement the active portfolio strategy that optimally exploits predictability. The question is, how much of their expected return would the investor be willing to give up (e.g. pay as a management fee) in return for having access to the superior strategy? Put differently, by how much does the return on the inferior strategy have to be increased to make the investor indifferent between the optimal and the inferior strategy. A precise definition of this premium is given in Appendix A.3. 


\section{Empirical Analysis}

We estimate a linear predictive model of the form,

$$
\left(r_{t}^{k}-r_{t-1}^{f}\right)=\left(\bar{\mu}_{k}-r_{t-1}^{f}\right)+B_{k} \cdot Z_{t-1}+\varepsilon_{t}^{k},
$$

where $Z_{t-1}$ is the vector of (lagged) predictive instruments, and $r_{t}^{k}$ are the returns on the risky assets. We assume that the residuals $\varepsilon_{t}^{k}$ are serially independent and independent of $Z_{t-1}$. This implies that the conditional variance-covariance matrix $\Sigma$ does not depend on $Z_{t-1}$. However, because we will estimate (1) jointly across all assets, we do not assume the $\varepsilon_{t}^{k}$ to be cross-sectionally uncorrelated, i.e. we do not assume $\Sigma$ to be diagonal.

Tables 1 and 2 show the in-sample estimation results, using the market index (Table 1) as well as the momentum portfolios (Table 2) as base assets. In each table, Column (1) reports the results using only business cycle variables as predictors, while Column (2) includes all instruments. While business cycle variables seem to possess very little predictive power (explaining only about $1 \%$ of the variation in the market index), the $R^{2}$ increases to about $6 \%$ when the sentiment variables are added. This is also reflected in the theoretically maximum Sharpe ratios, which are increased only marginally (from 0.49 to 0.61 in the case of the momentum portfolios) by the use of macro indicators, the increase not being statistically significant (with a $p$-value of almost 0.4 ). In contrast, the addition of sentiment indicators more than doubles Sharpe ratios (to 0.98 and 1.16, respectively). This increase is statistically significant (at the $5 \%$ level in the case of market-timing, and at the $1 \%$ level for momentum strategies). The benefit of adding sentiment to the set of predictive variables is also illustrated in Figure 3, which shows the efficient frontiers with and without predictability. Clearly, macro variables enlarge the investor's opportunity set only marginally (Panel A), while the addition of sentiment indicators has a much more dramatic effect (Panels B and C). Finally, while allowing the investor to pick between winner and loser portfolios does improve performance, this increase is much less dramatic. 


\subsection{Do Sentiment Indicators Protect Against Crashes?}

To begin, we conduct several out-of-sample experiments, focusing on times of high volatility or extreme market movements. We estimate the predictive model using data up the month preceding the event in question, and then study the performance of the resulting dynamically managed portfolio strategy during the following months.

\section{OCtober 1987 Stock Market Crash}

In our first experiment, we focus on the October 1987 market crash. Panel (A) of Figure 1 shows the cumulative returns of the market index and a dynamically optimal market-timing strategy, using only the business cycle variables as predictors. The bottom graph in Panel (A) shows the portfolio weights of the market-timing strategy. Although the market-timing strategy loses much less than the market index in the two months following the crash, it is evident that macro indicators alone cannot provide full insurance against the crash.

Panel (B) of Figure 1 shoes the performance of the market-timing strategy when the sentiment indicators are added to the set of predictors. First we note that the strategy in this case only loses about $5 \%$ of its value (in contrast to a loss of about $15 \%$ for the macrostrategy). Second, the strategy recovers much more quickly than both the market index and the macro-strategy. The bottom part of the figure shows that the sentiment-strategy achieves its performance by 'cutting its losses' by shorting the index just after the crash. In contrast, the macro-strategy remains heavily invested in the index throughout.

Finally, Panel (C) of Figure 1 shows the performance of the optimal strategy in the case where the investor is allowed to not only time the market but also allocate between 'winner' and 'loser' portfolios. This strategy incurs virtually no losses around the crash. Interestingly, while the market-timing strategy was forced to trade out of the market, the momentumstrategy achieves its superior portfolio insurance simply by trading on the spread between winners and losers, while remaining almost fully invested in the risk-free asset. 
Collapse of The 'DOT.COM' BubBle In 2000

we repeat the above experiment, this time focusing on the collapse of the internet bubble in late 2000. The results are shown in Figure [2]. From Panel (A) we see that the market-timing strategy based on business cycle variables alone performs only very marginally better than the market index itself. This is largely due to the fact that the strategy is very heavily invested in the index, indicating that the macro instruments fail to predict the bear market. In contrast, the inclusion of sentiment indicators (Panel B) dramatically improves the portfolio insurance aspect of the strategy. The portfolio weights show a similar pattern as around the 1987 crash, moving out of the market during the periods of sharpest decline. Finally, the strategy that uses winner and loser portfolios incurs virtually no losses throughout the entire bear market. In fact, although designed to minimize volatility, the portfolio gains $50 \%$ by the end of 2003. Relative to the market index, the sentiment-strategy achieves an annual alpha of almost $16 \%$, while the market-timing strategy based on macro instruments has an alpha of only $2 \%$. In contrast to the case of the 1987 crash, the momentum-strategy now keeps its investment in winner stocks virtually constant at $40 \%$, while timing the losers against the risk-free asset. Interestingly, the strategy invests most heavily in the loser portfolio during the recovery period in 2003.

In line with the findings of our in-sample analysis (see following Section), we find that strategies driven by business cycle variables are, unsurprisingly, high beta strategies. In contrast, sentiment indicators allow the portfolio manager to de-couple their portfolios from the market index (with betas no higher than about 0.65 ).

\subsection{Long-Run Portfolio Performance}

Next we investigate the long-run performance of the different types of strategies. The results are reported in Tables 3 and 4. First we note that the ex-post performance of all strategies matches closely the theoretical predictions, their Sharpe ratios coinciding with the theoretical ones reported in Tables 1 and 2. This is also illustrated in Figure 3, which plots the ex-post 
performance of the dynamically optimal minimum-variance and maximum-return strategies in relation to the theoretical efficient frontier.

\section{MARKet Timing}

Focusing first on pure market-timing strategies (Table 3), we find again evidence for the incremental benefit of adding sentiment variables to the set of predictors. For example, while both minimum-variance strategies (Panel A) track their target mean of $15 \%$ very closely, using sentiment indicators reduces volatility by almost $50 \%$ (from $13.1 \%$ to $7.8 \%$ ). The ability of sentiment indicators to help manage portfolio risk is illustrated in Figure 5, which shows the total return on the two minimum-variance strategies in relation to the market index. In particular in the period around the crash of the 'dot.com' bubble in 2000, the strategy based on business cycle variable tracks the index closely, showing extreme volatility and incurring losses in excess of $25 \%$. In contrast, the sentiment-based strategy is much less volatile and provides effective insurance against the dramatic market decline between 2000 and 2002. From Table 3 we see that a risk-averse investor (we used a moderate coefficient of risk aversion of 5) would be willing to give up 6.7 percentage points of annual return as management fee for the optimal strategy.

Similarly, the maximum-return strategies (Panel B) both match their volatility target of $15 \%$, but the strategy based on sentiment achieves an average return of $22.3 \%$, almost 700 basis points more than the macro-strategy. The superior market-timing ability of the sentiment strategy is illustrated in Figure 4, which shows the total return on the two maximum-return strategies in relation to the market index. While the strategy based on business cycle indicators under-performs the index throughout more than half of the sample period, the sentiment-based strategy not only consistently out-performs the market index by a wide margin, but also avoids losing value during the October 1987 crash and the collapse of the internet bubble. The strategy achieves an incredible alpha of $12.5 \%$ (as opposed to $3.6 \%$ for the macro-strategy), while its volatility is in fact lower than that of the index itself. An investor with a risk aversion coefficient of 5 would be willing to give up 9.0 percentage points of annual return as management fee for this strategy. 


\section{Momentum Strategies}

Allowing the investor in addition to pick between 'winner' and 'loser' portfolios further improves portfolio performance (Table 4), although the increase is much less dramatic.

Strategies based on these variables (even when allowed to pick between winners and losers) simply track the index with betas in excess of 1, thus amplifying any market movement. In contrast, the addition of sentiment indicators leads to significantly higher performance (with alphas increasing from 3-5\% to more than 15\%). For the same target return, efficient risk-control strategies based on business cycle variables still have a volatility of $14 \%$, while those using sentiment decrease volatility to about 7\%. Interestingly however, strategies based only on sentiment do not perform well at all. It is the interaction between business cycle indicators and sentiment that makes the strategies work.

\subsection{How do the Strategies Work?}

First, we note (Tables 3 and 4) that the strategies based on sentiment (even without allowing for momentum investing) tend to have much lower betas (between 0.1 and 0.3 ) than those based on business cycle variables alone. In other words, macro-strategies are effectively 'index-trackers', their high betas amplifying market movements in either direction. Thus, these are 'fair-weather-strategies', performing well in bull markets but incurring excessive losses during market downturns. In contrast, sentiment indicators seem to allow asset managers to 'de-couple' their portfolios from the business cycle and thus successfully time the market.

When we move to momentum strategies, we also find an interesting difference in the behavior of portfolio weights. While market-timing strategies are forced to move in and out of the market, often taking short or leveraged positions, we find that momentum strategies behave much more like hedge funds: in periods of market turmoil, they remain fully invested in the risk-free asset while taking spread trades (i.e. long-short positions) in the momentum 
portfolios. In bull markets, they remain heavily invested in the winner stocks, while taking spread positions between losers and the risk-free asset.

\section{What CAN We Learn From Investor Sentiment?}

An inspection of the coefficients on the sentiment variables (Table 1) shows that bullish investor sentiment $(N B>0)$ actually represents bad news: in the optimal market-timing strategy, the weight on the market index is negatively correlated with NB. In other words, "when the majority of investors predict economic conditions to improve, get out of the market!" This result is consistent with the over-confidence hypothesis (Shefrin 1999). For momentum-strategies, we find a similar pattern: in the optimal strategies, NB is negatively correlated with the weight on the 'winner' portfolio, and positively correlated with 'losers'.

We also find that $N B-N G$ is negatively correlated with the weights on 'winners', and positively correlated with 'losers'. In other words, "if business conditions are perceived to be bad today but expected to get better, short winner stocks and buy the losers." This result supports the under-reaction hypothesis (i.e. the loser stocks are those whose prices have not yet adjusted to the good news).

Finally, we find that $N B 3 L-N G$ is is negatively correlated with the weights on 'winners', and positively correlated with 'losers'. In other words, "if investors did expect business conditions to improve but this expectation has not been met, short winner stocks and buy the losers." This result supports the over-reaction hypothesis (i.e. the winner stocks are those whose prices over-reacted to the good news and are now over-valued).

\section{Does Sentiment Predict the Business Cycle?}

There is no evidence that sentiment is a reliable predictor of the business cycle. In fact, positive investor outlook is negatively correlated with future market movements. Our results show that macro-economic variables alone improve portfolio performance only marginally, while the addition of sentiment indicators has a much more dramatic effect. In other words, investor sentiment clearly contains information beyond simply predicting the business cycle. 
On the other hand, if we remove the macro-economic variables from the set of predictors, the performance of our strategies deteriorates dramatically. In other words, it is the interaction between traditional business cycle predictors and the sentiment variables that make our strategies work. Neither set of variables achieves any significant performance gains on its own.

\section{Conclusions}

Recent finance research that draws on behavioral psychology suggests that that investors systematically make errors in forming expectations about asset returns, and thus that investor sentiment can have predictive power for asset returns. A number of empirical studies using both market and survey data as proxies for investor sentiment have found support for these theories. In this study we investigate whether investor sentiment as measured by a component of the University of Michigan survey can help improve dynamic asset allocation over and above the improvement achieved based on commonly used business cycle indicators.

We find that sentiment-based dynamic trading strategies, even out-of-sample, would not have incurred any significant losses during the October 1987 crash or the collapse of the 'dot.com' bubble in late 2000. In contrast, standard business cycle indicators fail to predict these events, so that investors relying on these variables alone would have incurred significant losses. Interestingly however, strategies based only on sentiment do not perform well at all. It is the interaction between business cycle indicators and sentiment that makes the strategies work. An in-sample test shows that these results are statistically significant and not just artifacts of the chosen sub-period. Finally, the strategies based on the sentiment variables are 'active alpha' strategies with low betas and high alphas, in contrast to business cycle based strategies which are effectively 'index-trackers' with high betas with considerably lower alphas. 


\section{References}

Abhyankar, A., D. Basu, and A. Stremme (2005): "The Optimal Use of Asset Return Predictability: An Empirical Analysis," working paper, Warwick Business School.

Amromin, G., and S. Sharpe (2005): "From the Horse's Mouth: Gauging Conditional Expected Stock Returns from Investor Survey," working paper, Federal Reserve Board, Washington D.C.

Barberis, N., A. Shleifer, and R. Vishny (1998): "A Model of Investor Sentiment," Journal of Financial Economics, 49, 307-343.

Breen, W., L. Glosten, and R. Jagannathan (1989): "Economic Significance of Predictable Variation in Stock Index Returns," Journal of Finance, 44, 1177-1189.

Charoenrook, A. (2005): "Does Sentiment Matter?," working paper, Vanderbilt University.

Cochrane, J. (1999): "Portfolio Advice for a Multi-Factor World," Economic Perspectives, Federal Reserve Bank of Chicago, 23(3), 59-78.

Daniel, K., D. Hirshleifer, and A. Subrahmanyam (2001): "Overconfidence, Arbitrage, and Equilibrium Asset Pricing," Journal of Finance, 56, 921-965.

DeBondt, W., and R. Thaler (1985): "Does the Stock Market Overreact?," Journal of Finance, 40, 793-805.

Edwards, W. (1968): "Conservatism in Human Information Procession," in Formal Representation of Human Judgment, ed. by B. Kleimnutz, pp. 17-52. John Wiley \& Sons.

FAmA, E., and K. French (1988): "Dividend Yields and Expected Stock Returns," Journal of Financial Economics, 22, 3-25.

FAma, E., and G. Schwert (1977): "Asset Returns and Inflation," Journal of Financial Economics, 5, 115-146. 
Ferson, W., and A. Siegel (2001): "The Efficient Use of Conditioning Information in Portfolios," Journal of Finance, 56(3), 967-982.

Fisher, K., and M. Statman (2000): "Investor Sentiment and Stock Returns," Financial Analysts Journal, 56(2), 16-23.

Fleming, J., C. Kirby, and B. Ostdiek (2001): "The Economic Value of Volatility Timing," Journal of Finance, 56(1), 329-352.

Hirshleifer, D. (2001): "Investor Psychology and Asset Pricing," Journal of Finance, $56(4), 1531-1597$.

Jagannathan, R. (1996): "Relation between the Slopes of the Conditional and Unconditional Mean-Standard Deviation Frontier of Asset Returns," in Modern Portfolio Theory and its Applications: Inquiries into Asset Valuation Problems, ed. by S. Saito et al. Center for Academic Societies, Osaka, Japan.

Jegadeesh, N., and S. Titman (1993): "Returns to Buying Winners and Selling Losers: Implications for Stock Market Efficiency," Journal of Finance, 48, 65-91.

Kahneman, D., and A. Tversky (1973): "On the Psychology of Prediction," Psycological Review, 80, 237-251.

Kandel, S., and R. Stambaugh (1996): "On the Predictability of Stock Returns: An Asset Allocation Perspective," Journal of Finance, 51(2), 385-424.

Shefrin, H. (1999): Beyond Greed and Fear: Understanding Behavioral Finance and the Psychology of Investing. Oxford University Press, Oxford.

Shiller, R. (2000): Irrational Exuberance. Princeton University Press, Princeton, NJ.

ShleIfer, A. (2000): Inefficient Markets. Oxford University Press, Oxford.

Whitelaw, R. (2005): "Time Varying Sharpe Ratios and Market Timing," working paper, New York University. 


\section{A Appendix}

\section{A.1 Dynamically Efficient Strategies}

To specify a dynamically managed trading strategy, we denote by $\theta_{t-1}^{k}=\theta^{k}\left(Z_{t-1}\right)$ the fraction of portfolio wealth invested in the $k$-th risky asset at time $t-1$, given as a function of the vector $Z_{t-1}$ of (lagged) predictive instruments. The return on this strategy is given by,

$$
r_{t}(\theta)=r_{t-1}^{f}+\sum_{k=1}^{n}\left(r_{t}^{k}-r_{t-1}^{f}\right) \theta_{t-1}^{k},
$$

where $r_{t}^{k}$ is the return on the $k$-th risky asset, and $r_{t-1}^{f}$ denotes the return on the risk-free Treasury bill. The difference in time indexing indicates that, while the return $r_{t-1}^{f}$ on the risk-free asset is known at the beginning of the period, the returns $r_{t}^{k}$ on the risky assets are uncertain ex-ante and only realized at the end of the period. Note however that we do not assume $r_{t-1}^{f}$ to be unconditionally constant. It can be shown ${ }^{6}$ that the weights of any unconditionally efficient managed strategy can be written as,

$$
\theta_{t-1}^{*}=\frac{w-r_{t-1}^{f}}{1+H_{t-1}^{2}} \cdot \Sigma_{t-1}^{-1}\left(\mu_{t-1}-r_{t-1}^{f} e\right)
$$

Here, $\mu_{t-1}$ and $\Sigma_{t-1}$ are the conditional (on $Z_{t-1}$ ) mean vector and variance-covariance matrix of the base asset returns, and $w \in \mathbb{R}$ is a constant. By choosing $w \in \mathbb{R}$ in (3) appropriately, we can construct efficient strategies that track a given target expected return or target volatility.

\section{A.2 Measures of Statistical Significance}

To measure the economic gain due to predictability, we measure the extent to which the optimal use of predictive information expands the unconditionally efficient frontier, i.e. the

\footnotetext{
${ }^{6}$ See for example Ferson and Siegel (2001), or Abhyankar, Basu, and Stremme (2005).
} 
opportunity set available to the investor. In the absence of an unconditionally risk-free asset, the efficient frontier is described by three parameters, the location (mean and variance) of the GMV, and the asymptotic slope of the frontier (i.e. the maximum Sharpe ratio relative to the zero-beta rate corresponding to the mean of the GMV). Note however that because of the low volatility of T-bill returns, the location of the GMV will be virtually unaffected by the introduction of predictive instruments (see also Figure 3). Therefore, we focus here on the change in asymptotic slope of the frontier as a measure of predictability. Denote by $\lambda_{*}$ the slope of the frontier with optimal use of predictability, and by $\lambda_{0}$ the slope in the fixed-weight case (without making use of predictive information). In a slight abuse of terminology, we often refer to $\lambda_{*}$ and $\lambda_{0}$ simply as Sharpe ratios.

One can now show ${ }^{7}$ that up to a first-order approximation, the (squared) maximum slope of the dynamically managed frontier is given by,

$$
\lambda_{*}^{2} \approx E\left(H_{t-1}^{2}\right), \quad \text { where } \quad H_{t-1}^{2}=\left(\mu_{t-1}-r_{t-1}^{f} e\right)^{\prime} \Sigma_{t-1}^{-1}\left(\mu_{t-1}-r_{t-1}^{f} e\right) .
$$

Here, $\mu_{t-1}$ and $\Sigma_{t-1}$ are the conditional mean vector and variance-covariance matrix of the base asset returns. The error in the above approximation is of the order $\operatorname{var}\left(H_{t-1}^{2}\right)$. To obtain the corresponding expression for $\lambda_{0}$, we simply replace $\mu_{t-1}$ and $\Sigma_{t-1}$ by their unconditional counterparts.

Note that $H_{t-1}$ is the conditional Sharpe ratio, once the realization of the conditioning instruments is known. From (3), it is clear that $H_{t-1}$ plays a key role in the behavior of the optimal strategy. Moreover, the above result shows that the maximum unconditional Sharpe ratio is given by the unconditional second moment of the conditional Sharpe ratio ${ }^{8}$. Consequently, time-variation in the conditional Sharpe ratio improves the ex-post risk-return trade-off for the mean-variance investor, a point also noted by Cochrane (1999).

To measure the effect of predictability, we define the test statistic $\Omega=\lambda_{*}^{2}-\lambda_{0}^{2}$. Our null

\footnotetext{
${ }^{7}$ See, for example, Abhyankar, Basu, and Stremme (2005).

${ }^{8}$ In the case of a single risky asset, this was shown by Jagannathan (1996).
} 
hypothesis is that predictability does not matter, i.e. $\Omega=0$. As the set of fixed-weight strategies is contained in the set of dynamically managed strategies, we always have $\Omega \geq 0$. In the linear predictive setting (1) used in our empirical analysis, one can show ${ }^{9}$ that under the null, the test statistic

$$
\frac{T-K-1}{K} \cdot \Omega \text { is distributed as } F_{K, T-K-1} \text { in finite samples, }
$$

and $T \cdot \Omega$ is distributed as $\chi_{K}^{2}$ asymptotically. Here, $K$ is the number of instruments in $Z_{t-1}$, and $T$ is the number of time-series observations. This result allows us to assess the statistical significance of the economic gains due to predictability.

\section{A.3 Measures of Economic Value}

In addition to our statistical tests, we also employ a utility-based framework to assess the economic value of return predictability. Following Fleming, Kirby, and Ostdiek (2001), we consider a risk averse investor whose preferences over future wealth are given by a quadratic von Neumann-Morgenstern utility function. They show that, if relative risk aversion $\gamma$ is assumed to remain constant, the investor's expected utility can be written as,

$$
\bar{U}=W_{0}\left(E\left(r_{t}\right)-\frac{\gamma}{2(1+\gamma)} E\left(r_{t}^{2}\right)\right)
$$

where $W_{0}$ is the investor's initial wealth and $r_{t}$ is the return on the portfolio they hold. Consider now an investor who faces the decision whether or not to acquire the skill and/or information necessary to implement the active portfolio strategy that optimally exploits predictability. The question is, how much of their expected return would the investor be willing to give up (e.g. pay as a management fee) in return for having access to the superior strategy? To solve this problem, we need to find the solution $\delta$ to the equation

$$
E\left(r_{t}^{*}-\delta\right)-\frac{\gamma}{2(1+\gamma)} E\left(\left(r_{t}^{*}-\delta\right)^{2}\right)=E\left(r_{t}\right)-\frac{\gamma}{2(1+\gamma)} E\left(r_{t}^{2}\right),
$$

\footnotetext{
${ }^{9}$ See for example Abhyankar, Basu, and Stremme (2005).
} 
where $r_{t}^{*}$ is the optimal strategy and $r_{t}$ is a fixed-weight strategy that does not take predictability into account. The solution $\delta$ represents the management fee (as a fraction of portfolio returns) that the investor would be willing to pay in order to gain access to the superior strategy. Graphically, the premium can be found in the mean-variance diagram by plotting a vertical line downwards, starting from the point that represents the optimal strategy $r_{t}^{*}$, and locating the point where this line intersects the indifference curve through the point that represents the inferior strategy $r_{t}$. 
Panel (A)

Market-Timing (Macro Instruments)
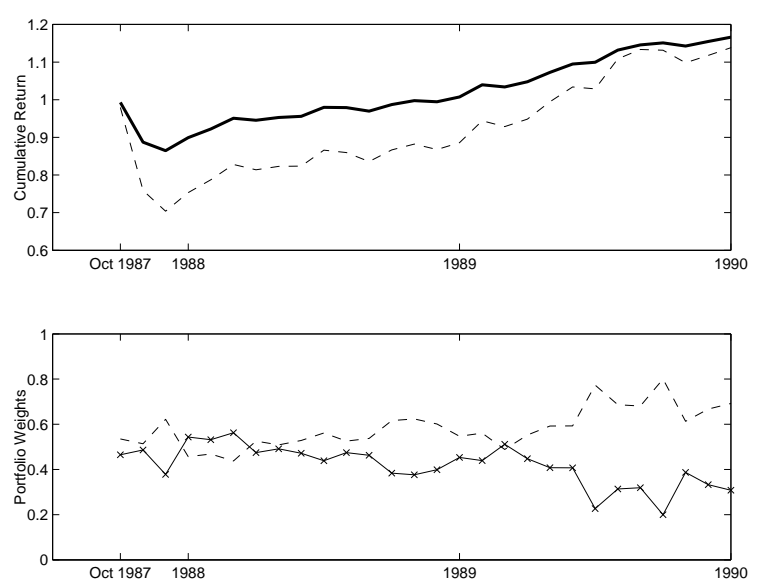

Panel (B)

Market-Timing (All Variables)
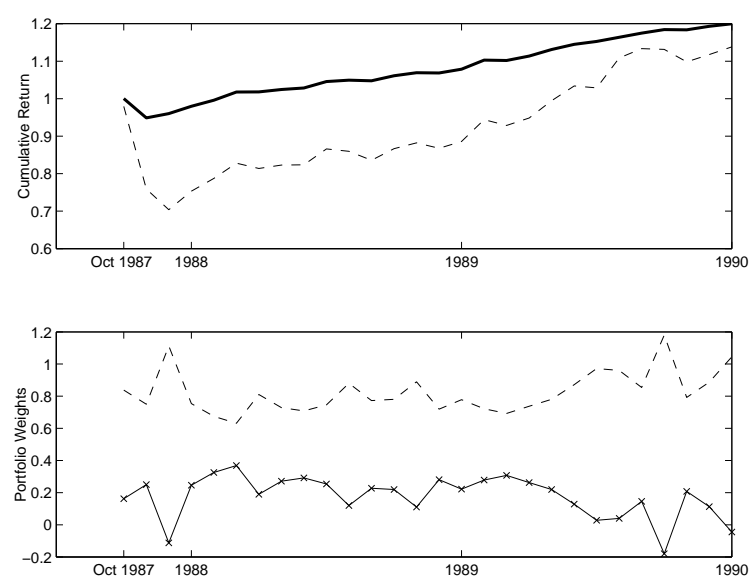

Panel $(\mathrm{C})$

Momentum-Strategy (All Variables)
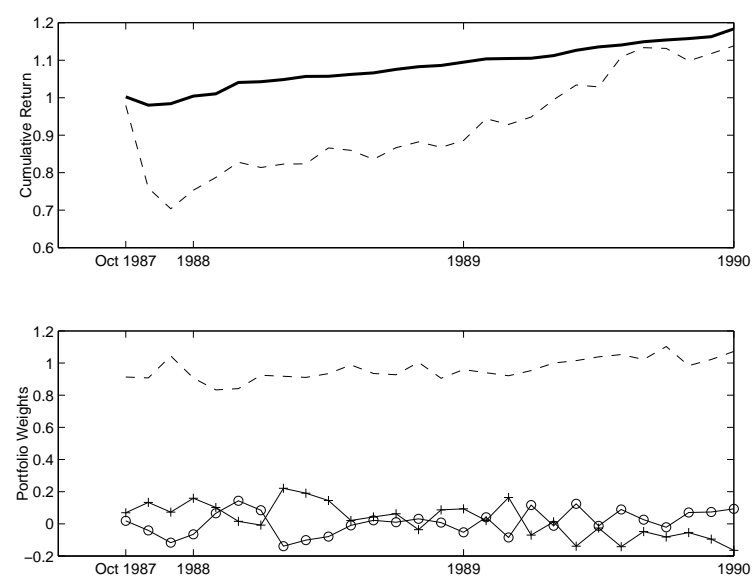

Figure 1: October 1987 Crash

These graphs show the performance and portfolio weights of three different strategies around the October 1987 market crash. In each panel, the top graph shows the cumulative return of the strategy (solid line) and the market index (dashed line), normalized to have unit value in September 1987. The bottom graph shows the portfolio weights on the risk-free asset (dashed line), the market index (' $\times$ '), or the winners ('+') or losers ('o') portfolios, respectively. Panels (A) and (B) focus on market-timing (i.e. allocating between the risk-free asset and the index), while Panel $(\mathrm{C})$ allows in addition allocation between the two momentum (winners and losers) portfolios. The strategy in Panel (A) uses only macro (business cycle) instruments, while Panels (B) and (C) use all (including sentiment) instruments. 
Panel (A)

Market-Timing (Macro Instruments)
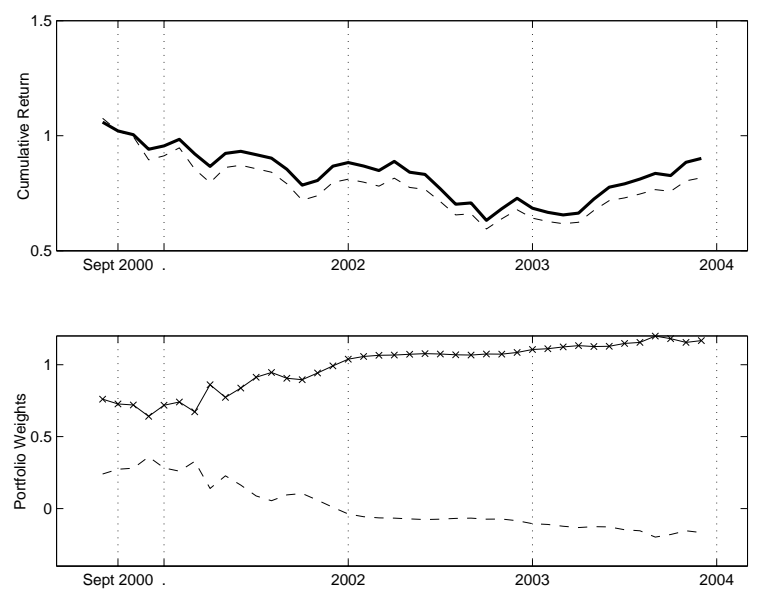

Panel (B)

Market-Timing (All Variables)
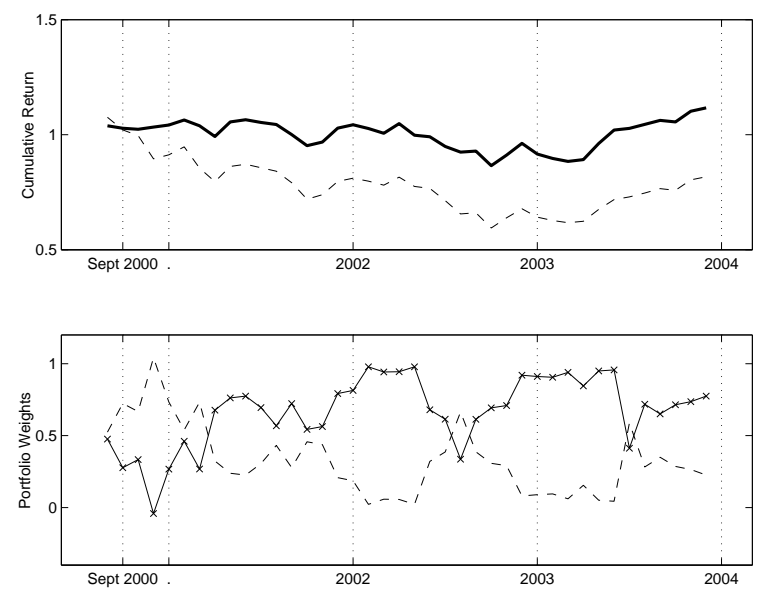

Panel (C)

Momentum-Strategy (All Variables)
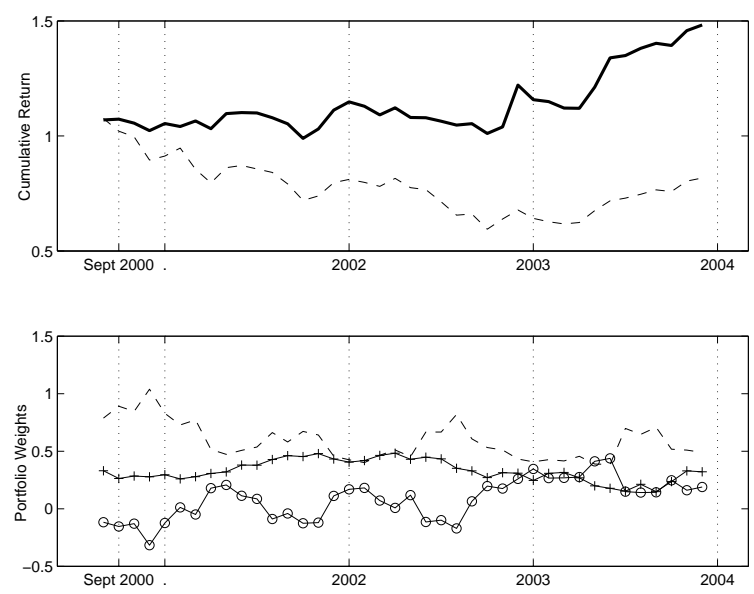

Figure 2: Collapse of the Internet Bubble

These graphs show the performance and portfolio weights of three different strategies around the collapse of the 'dot-com' bubble that began in late 2000. In each panel, the top graph shows the cumulative return of the strategy (solid line) and the market index (dashed line), normalized to have unit value in August 2000. The bottom graph shows the portfolio weights on the risk-free asset (dashed line), the market index (' $\times$ '), or the winners ('+') or losers ('o') portfolios, respectively. Panels (A) and (B) focus on market-timing (i.e. allocating between the risk-free asset and the index), while Panel (C) allows in addition allocation between the two momentum (winners and losers) portfolios. The strategy in Panel (A) uses only macro (business cycle) instruments, while Panels (B) and (C) use all (including sentiment) instruments. 


\begin{tabular}{|c|c|c|c|c|}
\hline \multirow{3}{*}{\multicolumn{2}{|c|}{ Panel (A) Summary Statistics }} & $\begin{array}{l}\text { Column (1) } \\
\text { ro Instruments }\end{array}$ & \multicolumn{2}{|c|}{$\begin{array}{l}\text { Column (2) } \\
\text { All Variables }\end{array}$} \\
\hline & & MKT & $\mathrm{RF}$ & MKT \\
\hline & & \multicolumn{3}{|c|}{ Panel (A) Summary Statistics } \\
\hline Average Return & $6.3 \%$ & $14.1 \%$ & $6.3 \%$ & $14.1 \%$ \\
\hline Volatility & $0.9 \%$ & $15.9 \%$ & $0.9 \%$ & $15.9 \%$ \\
\hline Sharpe Ratio & & 0.45 & & 0.45 \\
\hline \multicolumn{5}{|c|}{ Panel (B1) Regression Coefficients (Macro Instruments) } \\
\hline TB1M & & -2.75 & & -6.45 \\
\hline TSPR & & 0.75 & & 1.55 \\
\hline CSPR & & -0.85 & & -2.17 \\
\hline \multicolumn{5}{|c|}{ Panel (B2) Regression Coefficients (Sentiment Indicators) } \\
\hline NG & & & & -1.39 \\
\hline NB & & & & -0.28 \\
\hline NG3L & & & & 0.63 \\
\hline NB3L & & & & 0.33 \\
\hline NG6L & & & & 0.48 \\
\hline NB6L & & & & 0.33 \\
\hline$R^{2}$ & & $1.1 \%$ & & $5.8 \%$ \\
\hline \multicolumn{5}{|c|}{ Panel (C) Optimal Sharpe Ratio } \\
\hline Fixed-Weight & & & & \\
\hline Dynamically Managed & & & & \\
\hline (p-Value) & & & & \\
\hline
\end{tabular}

Table 1: Estimation Results (Market-Timing)

This table reports the summary of the full-sample estimates in the market-timing case. Column (1) reports the results when only business cycle variables are used, while in Column (2) all instruments (including sentiment indicators) are used. The table reports the mean-variance performance (Panel A) of the assets themselves, the coefficients of the predictive regression (Panel B), and the theoretically optimal Sharpe ratios (Panel C). The $p$-values are obtained from the asymptotic $\chi^{2}$-distribution of the test statistic $\Omega$ (see Appendix A.2). 


\begin{tabular}{|c|c|c|c|c|c|c|}
\hline \multirow{3}{*}{\multicolumn{2}{|c|}{ Panel (A) Summary Statistics }} & \multicolumn{2}{|c|}{$\begin{array}{c}\text { Column (1) } \\
\text { Macro Instruments }\end{array}$} & \multicolumn{3}{|c|}{$\begin{array}{l}\text { Column (2) } \\
\text { All Variables }\end{array}$} \\
\hline & & M01 & M10 & \multirow[t]{2}{*}{$\mathrm{RF}$} & \multirow[t]{2}{*}{ M01 } & \multirow[t]{2}{*}{ M10 } \\
\hline & & & & & & \\
\hline Average Return & $6.3 \%$ & $15.1 \%$ & $20.5 \%$ & $6.3 \%$ & $15.1 \%$ & $20.5 \%$ \\
\hline Volatility & $0.9 \%$ & $27.3 \%$ & $26.2 \%$ & $0.9 \%$ & $27.3 \%$ & $26.2 \%$ \\
\hline Sharpe Ratio & & 0.30 & 0.49 & & 0.30 & 0.49 \\
\hline \multicolumn{7}{|c|}{ Panel (B1) Regression Coefficients (Macro Instruments) } \\
\hline TB1M & & -2.70 & -2.80 & & -9.09 & -6.97 \\
\hline TSPR & & 0.58 & 0.25 & & 1.79 & 1.71 \\
\hline CSPR & & -0.69 & -0.17 & & -2.80 & -2.05 \\
\hline \multicolumn{7}{|c|}{ Panel (B2) Regression Coefficients (Sentiment Indicators) } \\
\hline NG & & & & & -1.60 & -2.74 \\
\hline $\mathrm{NB}$ & & & & & -1.52 & 0.27 \\
\hline NG3L & & & & & 0.67 & 2.13 \\
\hline NB3L & & & & & 0.92 & 0.25 \\
\hline NG6L & & & & & 0.30 & 0.40 \\
\hline NB6L & & & & & 0.63 & 0.22 \\
\hline$R^{2}$ & & $0.3 \%$ & $0.9 \%$ & & $5.9 \%$ & $4.6 \%$ \\
\hline \multicolumn{7}{|c|}{ Panel (C) Optimal Sharpe Ratio } \\
\hline Fixed-Weight & & 0.49 & & & 0.49 & \\
\hline Dynamically Managed & & 0.61 & & & 1.16 & \\
\hline ( $p$-Value) & & 0.381 & & & 0.002 & \\
\hline
\end{tabular}

\section{Table 2: Estimation Results (Momentum-Strategies)}

This table reports the summary of the full-sample estimates for the momentum portfolios. Column (1) reports the results when only business cycle variables are used, while in Column (2) all instruments (including sentiment indicators) are used. The table reports the mean-variance performance (Panel A) of the assets themselves, the coefficients of the predictive regression (Panel B), and the theoretically optimal Sharpe ratios (Panel C). The $p$-values are obtained from the asymptotic $\chi^{2}$-distribution of the test statistic $\Omega$ (see Appendix A.2). 
Panel (A)

Market-Timing (Macro Instruments)

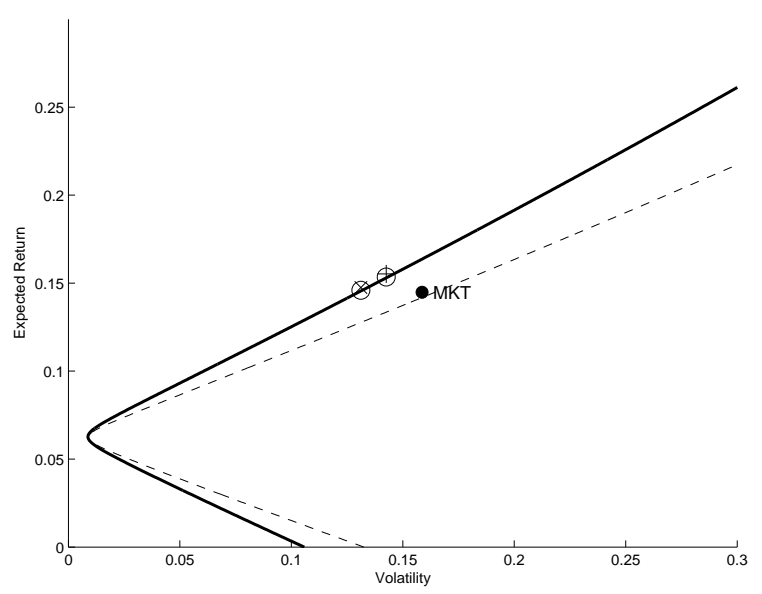

Panel (B)

Market-Timing (All Variables)

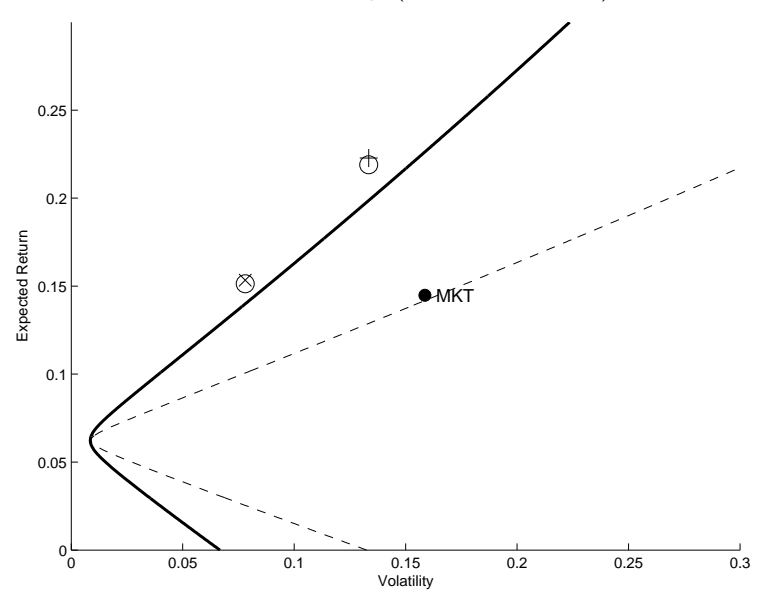

Panel (C)

Momentum-Strategy (All Variables)

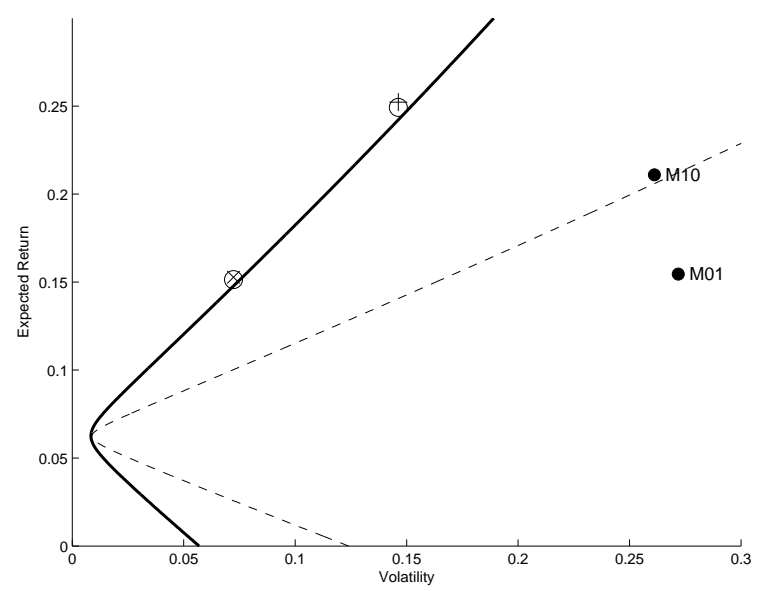

Figure 3: Efficient Frontiers

These graphs show the unconditionally efficient frontiers with (solid line) and without (dashed line) the optimal use of predictability. Panels (A) and (B) focus on market-timing (i.e. allocating between the risk-free asset and the index), while Panel (C) allows in addition allocation between the two momentum portfolios ('winners' M10 and 'losers' M01). The strategies in Panel (A) uses only macro (business cycle) instruments, while Panels (B) and (C) use all (including sentiment) instruments. Also shown are the ex-post mean and variance of the maximum-return (' + ') and minimum-variance (' $\times$ ') strategies. The circles indicate the performance of these strategies net of transaction costs. 


\begin{tabular}{|c|c|c|}
\hline \multirow{2}{*}{$\begin{array}{c}\text { Column (1) } \\
\text { Fixed-Weight }\end{array}$} & \multicolumn{2}{|c|}{ Column (2) } \\
\hline & \multicolumn{2}{|c|}{ Optimally Managed } \\
\hline & $\begin{array}{c}\text { Business Cycle } \\
\text { Instruments }\end{array}$ & $\begin{array}{l}\text { All Instruments } \\
\text { (incl. Sentiment) }\end{array}$ \\
\hline
\end{tabular}

Panel (A) Minimum-Variance Portfolio (Target Mean 15\%)

\begin{tabular}{lccc}
\hline & & \\
Expected Return & $14.9 \%$ & $14.8 \%$ & $15.3 \%$ \\
Volatility & $17.4 \%$ & $13.1 \%$ & $7.8 \%$ \\
Sharpe Ratio & 0.45 & 0.59 & 1.06 \\
CAPM Beta & 1.09 & 0.63 & 0.19 \\
Jensen's Alpha & $0.0 \%$ & $3.3 \%$ & $7.1 \%$ \\
Management Premium & & $3.3 \%$ & $6.7 \%$ \\
& & \\
Panel (B) Maximum-Return Portfolio (Target Volatility & $15 \%)$ & \\
& & & \\
Expected Return & $13.6 \%$ & $15.5 \%$ & $13.3 \%$ \\
Volatility & $14.9 \%$ & $14.3 \%$ & 1.06 \\
Sharpe Ratio & 0.45 & 0.59 & 0.33 \\
CAPM Beta & 0.94 & 0.68 & $12.5 \%$ \\
Jensen's Alpha & $0.0 \%$ & $3.6 \%$ & $9.0 \%$ \\
Management Premium & & $2.1 \%$ & \\
& & &
\end{tabular}

Table 3: Portfolio Performance (Market-Timing)

This table reports the ex-post performance of minimum-variance (Panel A) and maximum-return (Panel B) strategies, respectively. The available assets are the risk-free asset and the market index only. Column (1) reports the results for fixed-weight strategies (that do not use any predictive information), while Column (2) reports the results for optimally managed strategies using macro indicators or all predictive variables, respectively. All figures are annualized. 


\begin{tabular}{|c|c|c|}
\hline \multirow{2}{*}{$\begin{array}{l}\text { Column (1) } \\
\text { Fixed-Weight }\end{array}$} & \multicolumn{2}{|c|}{ Column (2) } \\
\hline & \multicolumn{2}{|c|}{ Optimally Managed } \\
\hline & $\begin{array}{l}\text { Business Cycle } \\
\text { Instruments }\end{array}$ & $\begin{array}{l}\text { All Instruments } \\
\text { (incl. Sentiment) }\end{array}$ \\
\hline
\end{tabular}

Panel (A) Minimum-Variance Portfolio (Target Mean 15\%)

\begin{tabular}{lccc}
\hline & & \\
Expected Return & $15.1 \%$ & $15.0 \%$ & $15.3 \%$ \\
Volatility & $16.2 \%$ & $13.1 \%$ & $7.2 \%$ \\
Sharpe Ratio & 0.49 & 0.62 & 1.14 \\
CAPM Beta & 0.86 & 0.50 & 0.13 \\
Jensen's Alpha & $1.9 \%$ & $4.5 \%$ & $7.6 \%$ \\
Management Premium & & $2.4 \%$ & $5.8 \%$ \\
& & \\
Panel (B) Maximum-Return Portfolio (Target Volatility & $15 \%)$ & \\
& & & \\
Expected Return & $14.3 \%$ & $16.3 \%$ & $14.6 \%$ \\
Volatility & $14.9 \%$ & $14.8 \%$ & 1.14 \\
Sharpe Ratio & 0.49 & 0.62 & 0.25 \\
CAPM Beta & 0.79 & 0.57 & $15.9 \%$ \\
Jensen's Alpha & $1.7 \%$ & $5.1 \%$ & $9.9 \%$ \\
Management Premium & & $1.7 \%$ & \\
& & &
\end{tabular}

Table 4: Portfolio Performance (Momentum-Strategy)

This table reports the ex-post performance of minimum-variance (Panel A) and maximum-return (Panel B) strategies, respectively. The available assets are the risk-free asset and the two (winners and losers) momentum portfolios. Column (1) reports the results for fixed-weight strategies (that do not use any predictive information), while Column (2) reports the results for optimally managed strategies using macro indicators or all predictive variables, respectively. All figures are annualized. 


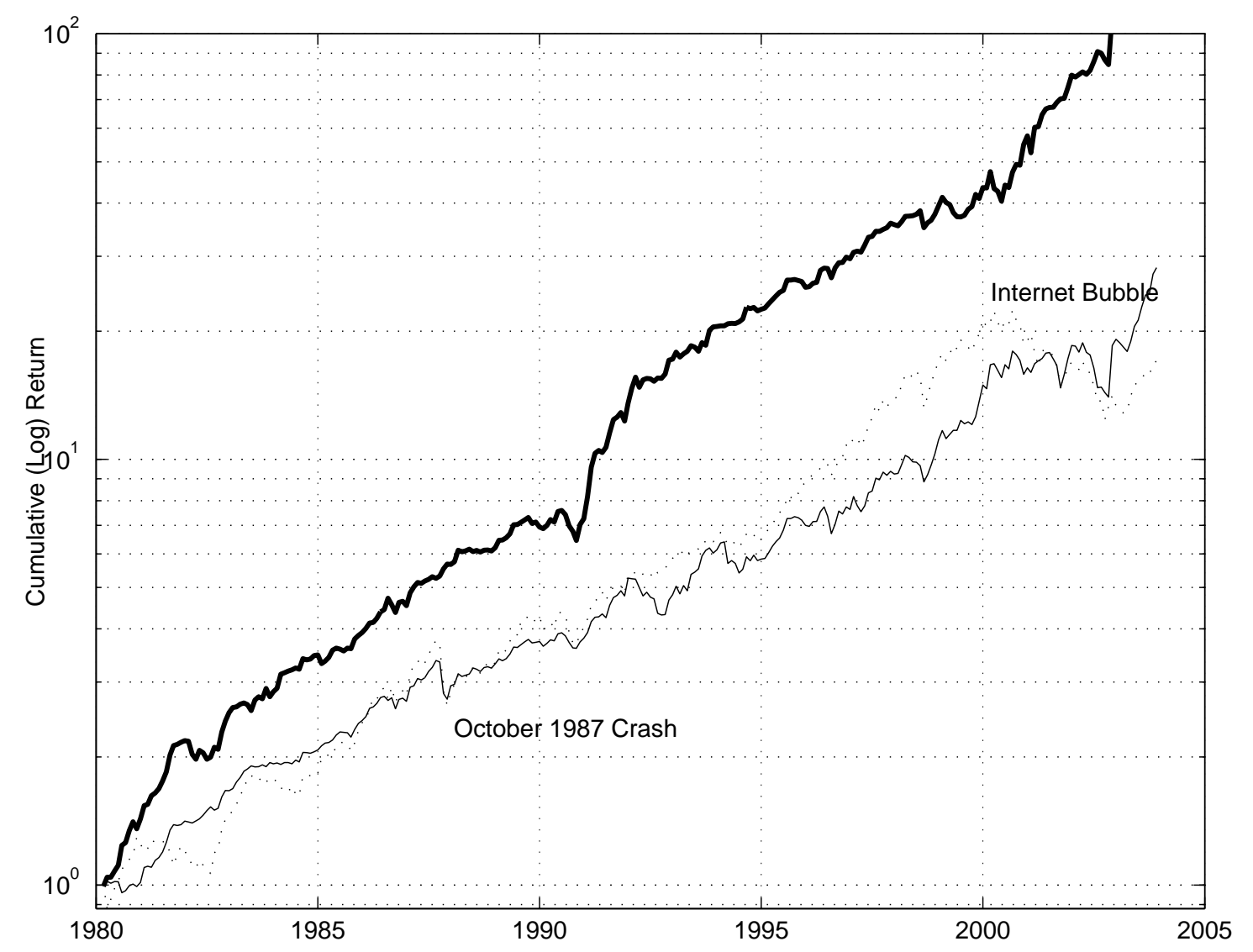

Figure 4: Total Return (Maximum-Return Strategy)

This figure shows the total cumulative (log) returns of the market index (dashed line), and the maximum-return market-timing strategies using macro variables only (light-weight line) and using all (including sentiment) instruments (bold-faced line). Portfolio values are normalized to unity in January 1980. Note that the vertical axis is logarithmic. 


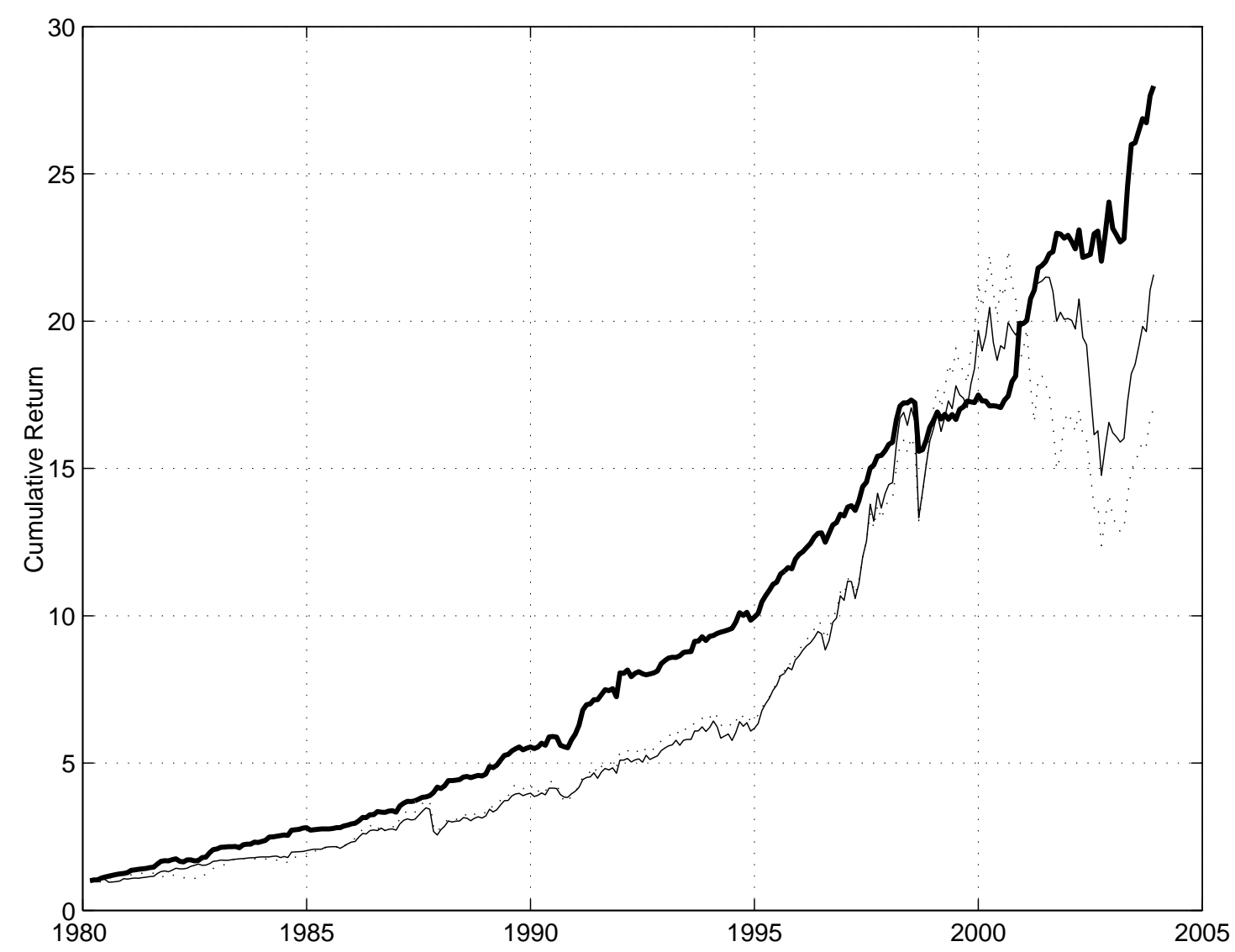

Figure 5: Total Return (Minimum-Variance Strategy)

This figure shows the total cumulative returns of the market index (dashed line), and the minimumvariance market-timing strategies using macro variables only (light-weight line) and using all (including sentiment) instruments (bold-faced line). Portfolio values are normalized to unity in January 1980. 